\title{
Prevalence and Influencing Factors on Fatigue of First-line Nurses Combating with COVID-19 in China: A Descriptive Cross-Sectional Study
}

\author{
Yu-xin ZHAN ${ }^{1 \dagger}$, Shi-yu ZHAO ${ }^{2 \dagger}$, Jiao YUAN ${ }^{3}$, Huan LIU ${ }^{4}$, Yun-fang LIU ${ }^{1}$, Ling-li GUI ${ }^{5}$, Hong ZHENG ${ }^{6}$, Ya-min ZHOU ${ }^{7}$, \\ Li-hua QIU ${ }^{8}$, Jiao-hong CHEN ${ }^{9}$, Jiao-hua YU", Su-yun LI ${ }^{1 \#}$ \\ ${ }^{I}$ Department of Nursing, Union Hospital, Tongji Medical College, Huazhong University of Science and Technology, Wuhan \\ 430022, China \\ ${ }^{2}$ Department of Operating Room, Union Hospital, Tongji Medical College, Huazhong University of Science and Technology, \\ Wuhan 430022, China \\ ${ }^{3}$ Department of Academic Research, Union Hospital, Tongji Medical College, Huazhong University of Science and Technology, \\ Wuhan 430022, China \\ ${ }^{4}$ Department of Nursing, Yijishan Hospital Affiliated to Wannan Medical College, Wuhu 241001, China \\ ${ }^{5}$ Department of Radiation and Medical Oncology, Zhongnan Hospital, Wuhan University, Wuhan 430071, China \\ ${ }^{6}$ Department of Urology, Renmin Hospital of Wuhan University, Wuhan 430060, China \\ ${ }^{7}$ Department of Operating Room, Affiliated Hospital of Jianghan University, Wuhan 430015, China \\ ${ }^{8}$ Department of Nursing, Union Jiangbei Hospital, Wuhan 430100, China \\ ${ }^{9}$ Department of Neurosurgery, Hubei Provincial Hospital of Integrated Chinese \& Western Medicine, Wuhan 430015, China
}

(C) Huazhong University of Science and Technology 2020

\begin{abstract}
Summary: Nurses' work-related fatigue has been recognized as a threat to nurse health and patient safety. The aim of this study was to assess the prevalence of fatigue among first-line nurses combating with COVID-19 in Wuhan, China, and to analyze its influencing factors on fatigue. A multi-center, descriptive, cross-sectional design with a convenience sample was used. The statistical population consisted of the first-line nurses in 7 tertiary general hospitals from March 3, 2020 to March 10, 2020 in Wuhan of China. A total of 2667 samples from 2768 contacted participants completed the investgation, with a response rate of $96.35 \%$. Social-demographic questionnaire, work-related questionnaire, Fatigue Scale-14, Generalized Anxiety Disorder-7, Patient Health Questionnaire-9, and Chinese Perceived Stress Scale were used to conduct online survey. The descriptive statistic of nurses' social-demographic characteristics was conducted, and the related variables of work, anxiety, depression, perceived stress and fatigue were analyzed by $t$-tests, nonparametric test and Pearson's correlation analysis. The significant factors which resulted in nurses' fatigue were further analyzed by multiple linear regression analysis. The median score for the first-line nurses' fatigue in Wuhan was $4(2,8)$. The median score of physical and mental fatigue of them was $3(1,6)$ and $1(0,3)$ respectively. According to the scoring criteria, $35.06 \%$ nurses $(n=935)$ of all participants were in the fatigue status, their median score of fatigue was $10(8,11)$, and the median score of physical and mental fatigue of them was $7(5,8)$ and $3(2,4)$ respectively. Multiple linear regression analysis revealed the participants in the risk groups of anxiety, depression and perceived stress had higher scores on physical and mental fatigue and the statistically significant positive correlation was observed between the variables and nurses' fatigue, the frequency of exercise and nurses' fatigue had a statistically significant negative correlation, and average daily working hours had a significantly positive correlation with nurses' fatigue, and the frequency of weekly night shift had a low positive correlation with nurses' fatigue $(P<0.01)$. There was a moderate level of fatigue among the first-line nurses fighting against COVID-19 pandemic in Wuhan, China. Government and health authorities need to formulate and take effective intervention strategies according to the relevant risk factors, and undertake preventive measures aimed at reducing health hazards due to increased work-related fatigue among first-line nurses, and to enhance their health status and provide a safe occupational environment worldwide. Promoting both medical and nursing safety while combating with the pandemic currently is warranted.

Key words: COVID-19; pandemic; Chinese nurses; fatigue; anxiety; depression; perceived stress; cross-sectional study
\end{abstract}

Yu-xin ZHAN, E-mail: 0109-kitty@163.com; Shi-yu ZHAO, E-mail: 648202059@qq.com

†The authors contributed equally to this work.

${ }^{\text {\#C} C o r r e s p o n d i n g ~ a u t h o r s, ~ J i a o-h u a ~ Y U, ~ E-m a i l: ~ y u j i a o h u a 2008 ~}$

@126.com; Su-yun LI, E-mail: lisuyun0503@163.com
Fatigue is a universal non-specific symptom, and it is not only an unwell reaction undernormal physiological conditions, but also the clinical manifestations of some diseases, meanwhile it is also a sub-health status between disease and non-disease, including 
physiological fatigue and psychological fatigue ${ }^{[1]}$. Nurse fatigue refers to a "work-related condition that ranges from acute to chronic in nature and can result in over-whelming sense of tiredness, decreased energy, and exhaustion, ultimately accompanied by impacting physical and cognitive functions"'[2]. Fatigue in nurses is complex and arises in response to individual, unit, and health care system demands ${ }^{[3]}$. Kahriman et al reported that $83 \%$ of medical errors were positively correlated with nurses' fatigue ${ }^{[4]}$.

The emergence of corona virus disease 2019 (COVID-19) in Wuhan, China was in December 2019 ${ }^{[5]}$. On March 11, 2020, the World Health Organization declared COVID-19 as a global pandemic with the spread of this worst global crisis ${ }^{[6]}$. All on-the-job nurses in Wuhan were fully engaged in the continuous combating with the prevention and control of the pandemic as the first-line nurses at the first time. The tremendous psychological burden and rescue challenge greatly aggravated the symptoms of fatigue among the first-line nurses in Wuhan, and easily led to various psychological abnormalities ${ }^{[7]}$. Previous study showed that there was a strong relationship between fatigue and the lifestyles and psychological states ${ }^{[8]}$. It was reported that a fall in resistance to the existing physical, mental and emotional stress in nurses might lead to anxiety, depression and ultimately fatigue $\mathrm{e}^{[9,10]}$. The sudden outbreak of SARS in 2003 had a psychological impact on nurses. Studies have shown that nurses are reluctant to go home for fear of infecting relatives, colleagues and friends ${ }^{[11-13]}$. Health care workers spent hours each day putting on and taking off airtight protective equipment, which aggravated the exhaustion that the workers were experiencing from the increasing workload with the outbreak of SARS ${ }^{[14]}$. The nurses' stress was found to be mainly due to the lack of protective gear and basic equipment, especially in the early phase of the Ebola virus disease outbreak ${ }^{[15]}$. In 2014, the Ebola outbreak was also a challenge for the physical and mental health of health care professionals. Despite shift hours and the risk of infection, the challenging treatment environment created anxiety, depression, fatigue and social isolation for health care professionals ${ }^{[16]}$. National Health Commission of China had published several guideline documents aimed to settle the emergency psychological crisis through establishing psychological assistance hotline and a series of interventions for the medial personnel during the pandemic. Furthermore, it would be necessary to carry out widespread surveillance to monitor and manage the risk of fatigue possibly involved in work-related and negative mental status among the first-line nurses.

At present, we found no large sample study on fatigue status and risk factors of the first-line nurses in Wuhan. The aim of this study is to evaluate the prevalence of fatigue status among first-line nurses responsible for rescue of COVID-19 in Wuhan, and to analyze the influencing factors associated with physical and mental fatigue of nurses. The findings of this study may be used to provide strategies to better assist health care administrators in addressing the physical and mental health of nurses domestically and internationally, and so as to supply references for the medical and health management to implement effective intervention to relieve the mental burden and reduce the fatigue level, contribute to construction of the safe occupational environment and promote medical and nursing safety.

\section{OBJECTS AND METHODS}

\subsection{Study Design and Participants}

A descriptive cross-sectional survey was conducted with a convenience sample of nurses from 7 hospitals in Wuhan, China from March 3 to 10, 2020. To be eligible, all participants were the registered nurses. All procedures performed in studies involving human participants were in accordance with the ethical standards of the institutional and/or national research committee and with the 1964 Helsinki declaration and its later amendments or comparable ethical standards. The including criteria for population included the nurses who have participated in the first-line nursing work of COVID-19 patients from January 1 till now. The nurses without nurse qualification certificate, foreign aid nurses, trainee nurses from other hospitals, and nurses who were continuously off duty from January 1 to the investigation period (maternity leave, sick leave, personal leave, etc.) were excluded from this study. According to the multi-factor analysis sample estimation method of 6-10 times variables of the total number of items in the questionnaire, the minimum sample size was 610 . Considering $20 \%$ of the invalid filling rate of the questionnaire, it was expected that at least 732 questionnaires would be conducted. Finally 2768 questionnaires were completed, and 2667 of them were valid, for a survey response rate of $96.35 \%$.

\subsection{Data Collection}

In this study, questionnaire stars were used to make two-dimensional code, and Wechat was used to distribute the questionnaire online. The research established a questionnaire survey group, which was mainly responsible for the distribution and collection of questionnaires in 7 hospitals respectively. After obtaining approval of ethics committee of the researcher's hospital and the consent and cooperation from 7 respective hospitals, each chief nurse in charge of hospitals was approached to be familiar with the purpose and procedure of the investigation. The researchers trained 7 investigators in a unified way, including the aim, composition and filling requirements of the questionnaire. The survey group checked the 
questionnaires one by one and eliminated the invalid ones. Exclusion criteria of invalid questionnaire were as follows: inconsistency in logic of answering, such as filling in contradictions and inconsistencies, answering time less than $300 \mathrm{~s}$, simple repetition and abnormal answering, and informed disagreement.

\subsection{Quality Control}

In order to ensure the accuracy of the data, the research group strictly implemented pre-control of the quality. (1) Questionnaire design: on the basis of consulting and referring to domestic and foreign literature, the scale was selected, modified and supplemented in combination with the opinions of public health management and nursing management experts to ensure the universality and specificity of the questionnaire; (2) Answer setting: all items were set as required questions to ensure the integrity of the questionnaire; the way of filling in the questionnaire was set as one time for each equipment answer to avoid repeated answers; (3) Pre-survey: the research designer, investigator and some respondents participated in the pre-survey of the questionnaire, and discussed and revised the design of the questionnaire and the setting of items. The revised questionnaire was used to carry out the pre-survey again to test the reliability of the method. (4) Formal investigation: convenient sampling method was adopted, and the trained investigators of each hospital used the guidance to fill in the instruction uniformly, so as to prevent investigation bias. The investigators used consistent language to explain when the respondents had questions. (5) Data processing: the data were encoded, input, summarized and checked by two members of the research group, and logical correction was carried out. Problems found were corrected in time, and invalid questionnaires were eliminated.

\subsection{Variables and Instruments}

1.4.1 Social-Demographic Questionnaire This questionnaire included general information about the age, gender, education level, years of working experiences, marital status, fertility status, professional titles, lifestyles within the last month such as average length of daily break and night sleep and exercise.

1.4.2 Work-Related Questionnaire This questionnaire asked information about the participants' current working status in hospital respectively.

1.4.3 Fatigue Scale-14 (FS-14) The scale was developed by King's College Hospital in the UK, which was mainly used to measure the severity of fatigue symptoms, evaluate clinical efficacy, and screen fatigue cases in epidemiological research ${ }^{[17]}$. There are 14 items in the scale which reflect the severity of fatigue from different perspectives: items 1-8 reflect physical fatigue, and items 9-14 reflect mental fatigue. The scores are added from items 1 to 8 to get the scores of physical fatigue, and the scores are added from items 9 to 14 to get the scores of mental fatigue, while the total score of fatigue is the sum of the scores of physical and mental fatigue. The highest physical fatigue score is 8 , the highest mental fatigue score is 6 , the highest total score is 14 , the total score $\geq 7$ implies that fatigue status exists. The higher the score, the more serious the reflection of fatigue. The total Cronbach's alpha coefficient is 0.7725 , and the half coefficient is 0.7725 , which indicates the scale has good internal consistency ${ }^{[18]}$. The Cronbach's alpha coefficient was 0.850 in our study.

\subsubsection{Generalized Anxiety Disorder-7 (GAD-7)}

The scale was developed by Spitzer et al in 2006 according to the diagnostic criteria of generalized anxiety disorder for screening of generalized anxiety disorder and evaluation of symptom severity ${ }^{[19]}$. The scale consists 7 items, each of which describes a typical symptom of generalized anxiety disorder. The scale is graded according to the status and frequency of its occurrence in the past two weeks. The total score is formed by adding the scores of each item of the scale, ranging from $0-21$. According to the scoring criteria, the results of scores are divided into four degrees: scores for 0-4, 5-9, 10-14 and 15-21, corresponding to none, mild, moderate and severe level of anxiety respectively ${ }^{[20]}$. The higher the score, the more serious the anxiety. GAD-7 is easy to implement, and has good reliability and validity in different populations. The Cronbach's alpha coefficient of GAD-7 in Chinese version is $0.898^{[21]}$. The Cronbach's alpha coefficient was 0.947 in our study.

\subsubsection{Patient Health Questionnaire-9 Item (PHQ-9)}

The scale was based on the nine criteria of depression in the Handbook of diagnosis and statistics of mental disorders published by the American Psychiatric Association ${ }^{[22]}$. The scale consists 9 items and the total score is composed of the scores of each item in the scale, ranging from 0 to 27 . According to the scoring criteria, the results of scores are divided into five degrees: score $0-4,5-9,10-14,15-19,20$ 27 , corresponding to none, mild, moderate, excessively moderate and severe level of depression respectively ${ }^{[23]}$. The higher the score, the more serious the depression. The Cronbach's alpha coefficient of PHQ-9 in Chinese version is $0.857^{[24]}$. The Cronbach's alpha coefficient was 0.933 in our study.

1.4.6 Chinese Perceived Stress Scale (CPSS) The scale was widely used in the mental health assessment of the occupational population. It was revised by Yang et al according to the foreign version of the perceived stress scale (PSS) into Chinese version ${ }^{[25]}$. The scale consists of 14 items reflecting stress tension and loss of control, and participants are required to answer according to their own feelings ${ }^{[26]}$. A score of 0-24 indicates normal pressure; $25-42$ indicates high pressure; 43-56 indicates excessive pressure. The 
scale has high homogeneity and internal consistency among different populations in China, and Cronbach's alpha coefficient is $0.797^{[25]}$. The Cronbach's alpha coefficient was 0.823 in our study.

\subsection{Statistical Analysis}

Data were analyzed using the IBM Statistical Package for Social Sciences (SPSS) version 23.0. Descriptive statistics were used to present participants' social-demographic and work-related factors and the prevalence of fatigue. Mann Whitney test and Kruskal Wallis test were used to analyze the fatigue status of nurses in different social-demographic characteristics, work-related variables, anxiety, depression and perceived stress levels; and Pearson's correlation analysis was conducted to examine the relationships between the anxiety, depression, perceived stress levels and fatigue of nurses; multiple stepwise linear regression analysis was performed to identify the influence of aforementioned variables on first-line nurses' fatigue. An alpha-level of $P<0.05$ was set for significance in all analyses.

\section{RESULTS}

2.1 Social-Demographic Characteristics of Participants

Of the 2667 nurses investigated in this study, $3.04 \%(n=81)$ were male, $96.96 \%(n=2586)$ were female; the median age was $30(26,35)$ years of age; the median year of working experiences was $8(4$, 13) years; $58.98 \%$ were married $(n=1573), 38.73 \%$ were unmarried $(n=1033), 2.29 \%$ were divorced and others $(n=61) ; 39.26 \%$ were unmarried and childless $(n=1047), 7.54 \%$ were married and childless $(n=201)$, $53.20 \%$ were married and childbearing $(n=1419)$. The characteristics of social-demographic are shown in table1.

\subsection{Descriptive Analyses of Prevalence of Physical and Mental Fatigue}

The median score of fatigue of first-line nurses was $4(2,8)$. The median score of physical fatigue was $3(1,6)$, the median score of mental fatigue was 1 $(0,3)$. Furthermore, $35.06 \%$ nurses $(n=935)$ of the all participants had a total score of $\geq 7$, the median score of them was $10(8,11)$, the median scores of physical and mental fatigue of participants with high level of fatigue were $7(5,8)$ and $3(2,4)$ respectively.

\subsection{Descriptive and Univariate Analyses of Factors} between Social-Demographic Variables and Fatigue

General data were used as independent variables and scores of physical fatigue and mental fatigue were used as dependent variables for nonparametric test. The results showed that age, years of working experiences, marriage and fertility status, average length of daily break and night sleep and exercise variables had an impact on the score of physical fatigue; years of working experiences, marriage and fertility status, average length of daily break and night sleep and exercise variables had an impact on the score of mental fatigue, and the difference was statistically significant $(P<0.05)$. The results are shown in table 1 .

\subsection{Descriptive and Univariate Analyses of Factors between Work-related Variables and Fatigue}

Work-related variables were used as independent variables and scores of physical and mental fatigue were used as dependent variables for nonparametric test. The results showed that the total number of days of rescue work, average daily working hours, frequency of night shifts, direct participation in the rescue of patients with COVID-19, professional protection training, professional psychological assistance, occupational exposure, infection with COVID-19, experience of negative events (the relatives, friends and colleagues of participants were seriously, critically ill or even dead due to the pandemic), and the degree of personal fear of COVID-19 variables had influence on the score of physical fatigue; the average daily working hours, frequency of night shifts, direct participation in the rescue of patients with COVID-19, professional protection training, occupational exposure, experience of negative events (the relatives, friends and colleagues of participants were seriously, critically ill or even dead due to the pandemic), and the degree of personal fear of COVID-19 variables could influence the score of mental fatigue, and the difference was statistically significant $(P<0.01)$. The results are shown in table 2 . 2.5 Descriptive, Univariate and Correlation Analyses between Anxiety, Depression, Perceived Stress Variables and Fatigue

Anxiety, depression and perceived stress were independent variables, and the scores of physical fatigue and mental fatigue were used as dependent variables for nonparametric test. 2667 nurses were divided into group of "yes" or "no" which indicated whether they were in risk of abnormal mental status or not, according to the scoring criteria of anxiety, depression and perceived stress scales respectively. The results showed that the total number of each risk group of anxiety, depression and perceived stress was 1062 (39.82\%), $1458(54.65 \%)$ and 1298 (62.00\%), meanwhile, the median scores of physical fatigue in each risk group of them were $6(4,8), 5(3,7)$ and 5 $(3,7)$, the median scores of mental fatigue in each risk group of them were $3(1,4), 2(1,4)$ and $3(1$, $4)$, respectively. The results showed that anxiety, depression and perceived stress could influence the scores of physical and mental fatigue. The difference was statistically significant $(P<0.01)$. The results of descriptive and univariate analyses of the factors are shown in table 3 , and those of the bivariate correlation analyses between anxiety, depression, perceived stress variables and fatigue are shown in table 4. 
Table 1 Descriptive and univariate analyses between social-demographic factors and fatigue ( $n=2667)$

\begin{tabular}{|c|c|c|c|c|c|}
\hline \multirow[b]{2}{*}{ Variables } & \multirow[b]{2}{*}{$n(\%)$} & \multicolumn{2}{|c|}{ Physical fatigue } & \multicolumn{2}{|c|}{ Mental Fatigue } \\
\hline & & M (P25, P75 ) & $\begin{array}{c}\mathrm{Z}^{*} / \mathrm{H}^{* *} \\
P\end{array}$ & $\mathrm{M}(\mathrm{P} 25, \mathrm{P} 75)$ & $\begin{array}{c}\mathrm{Z}^{*} / \mathrm{H}^{* *} \\
P\end{array}$ \\
\hline \multicolumn{6}{|l|}{ Gender } \\
\hline Male & $81(3.04)$ & $3(1,5.5)$ & $-1.081^{*}$ & $1(0,3)$ & $-1.543^{*}$ \\
\hline Female & $2586(96.96)$ & $3(1,6)$ & 0.279 & $2(0,3)$ & 0.123 \\
\hline \multicolumn{6}{|l|}{ Age groups (years) } \\
\hline$\leq 25$ & $655(24.56)$ & $3(1,5)$ & $27.897^{* *}$ & $1(0,3)$ & $7.847^{* *}$ \\
\hline $26-35$ & $1360(50.99)$ & $3(1,6)$ & $<0.001$ & $2(0,3)$ & 0.097 \\
\hline $36-45$ & $448(16.80)$ & $4(2,6)$ & & $1(0,3)$ & \\
\hline $46-55$ & $199(7.46)$ & $4(1,6)$ & & $1(0,3)$ & \\
\hline$\geq 56$ & $5(0.19)$ & $1(0.5,7.5)$ & & $1(0,3)$ & \\
\hline \multicolumn{6}{|l|}{ Working experiences (years) } \\
\hline$\leq 5$ & $1030(38.62)$ & $3(1,5)$ & $34.257^{* *}$ & $1(0,3)$ & $9.551^{* *}$ \\
\hline $6-10$ & $700(26.25)$ & $3(1,6)$ & $<0.001$ & $2(0,3)$ & 0.049 \\
\hline $11-15$ & $414(15.52)$ & $3(1,6)$ & & $2(0,3)$ & \\
\hline $16-20$ & $185(6.94)$ & $4(2,6)$ & & $1(0,3)$ & \\
\hline$\geq 21$ & $338(12.67)$ & $4(2,6)$ & & $1(0,3)$ & \\
\hline \multicolumn{6}{|l|}{ Marital status } \\
\hline Married & $1573(58.98)$ & $3(1,6)$ & $20.440^{* *}$ & $2(0,3)$ & $13.295^{* *}$ \\
\hline Unmarried & $1033(38.73)$ & $3(1,5)$ & $<0.001$ & $1(0,3)$ & 0.001 \\
\hline Divorced and others & $61(2.29)$ & $4(1,6)$ & & $2(0,3)$ & \\
\hline \multicolumn{6}{|l|}{ Fertility status } \\
\hline Unmarried and childless & 1047 (39.26) & $3(1,5)$ & $17.850^{* *}$ & $1(0,3)$ & $15.471^{* *}$ \\
\hline Married and childbearing & $1419(53.21)$ & $3(1,6)$ & $<0.001$ & $2(0,3)$ & $<0.001$ \\
\hline Married and childless & $201(7.54)$ & $3(1,6)$ & & $1(0,3)$ & \\
\hline \multicolumn{6}{|l|}{ Professional title } \\
\hline Nurse & $742(27.82)$ & $3(1,5)$ & $36.967^{* *}$ & $1(0,3)$ & $5.960^{* *}$ \\
\hline Secondary nurse & $1168(43.79)$ & $3(1,6)$ & $<0.001$ & $2(0,3)$ & 0.114 \\
\hline Chief nurse & $675(25.31)$ & $4(2,6)$ & & $2(0,3)$ & \\
\hline Deputy chief nurse and above & $82(3.07)$ & $4(2,7)$ & & $1(0,3)$ & \\
\hline \multicolumn{6}{|l|}{ Educational level } \\
\hline Technical secondary school & $22(0.82)$ & $2(1,5)$ & $5.717^{* *}$ & $0.5(0,3)$ & $7.394^{* *}$ \\
\hline Junior college & $569(21.33)$ & $3(1,6)$ & 0.126 & $1(0,3)$ & 0.060 \\
\hline Undergraduate course & $2039(76.45)$ & $3(1,6)$ & & $2(0,3)$ & \\
\hline Master's degree and above & 37 (1.39) & $3(1,4.5)$ & & $2(1,3)$ & \\
\hline \multicolumn{6}{|c|}{ Average length of daily break (minutes) } \\
\hline Never & $603(22.61)$ & $4(2,6)$ & $44.364^{* *}$ & $2(1,3)$ & $16.665^{* *}$ \\
\hline$<30$ & $853(31.98)$ & $3(1,6)$ & $<0.001$ & $2(0,3)$ & 0.001 \\
\hline $30-60$ & $1056(39.60)$ & $3(1,5)$ & & $1(0,3)$ & \\
\hline$>60$ & $155(5.81)$ & $2(1,4)$ & & $1(0,3)$ & \\
\hline \multicolumn{6}{|l|}{ Average length of daily sleep (hours) } \\
\hline$\leq 5$ & $164(6.15)$ & $5(3,7)$ & $64.876^{* *}$ & $3(1,4)$ & $23.801^{* *}$ \\
\hline $6-8$ & $2430(91.11)$ & $3(1,6)$ & $<0.001$ & $1(0,3)$ & $<0.001$ \\
\hline $9-10$ & $73(2.74)$ & $1(0,4)$ & & $1(0,3)$ & \\
\hline \multicolumn{6}{|c|}{ Average number of weekly exercise (times) } \\
\hline 0 & $1677(62.88)$ & $4(2,6)$ & $98.152^{* *}$ & $2(0,3)$ & $15.766^{* *}$ \\
\hline$\leq 1$ & $740(27.75)$ & $2(1,5)$ & $<0.001$ & $1(0,3)$ & 0.001 \\
\hline $2-3$ & $185(6.94)$ & $3(1,5)$ & & $1(0,3)$ & \\
\hline$\geq 4$ & $65(2.44)$ & $1(0,5)$ & & $1(0,3)$ & \\
\hline
\end{tabular}

M: median; P25: percentile 25; P75: percentile 75; ${ }^{*} \mathrm{Z}$ : Mann-Whitney test; ${ }^{* *} \mathrm{H}$ : Kruskal-Wallis test

2.6 Multiple Linear Regression Analyses of Fatigue with Work-related Factors, Anxiety, Depression and Perceived Stress Variables

Table 5 shows the correlations between the fatigue and work-related factors, anxiety, depression and perceived stress variables. The results of the regression indicated the predictors explained $53.1 \%$ of the variance $\left(R=0.730, R^{2}=0.534\right.$, Adjusted $R^{2}=0.531$, standard error of the estimate $=2.575, F=216.696, P<0.001)$. A statistically significant negative correlation was 
Table 2 Descriptive and univariate analyses between work-related variables and fatigue $(n=2667)$

\begin{tabular}{|c|c|c|c|c|c|}
\hline \multirow[b]{2}{*}{ Variables } & \multirow[b]{2}{*}{$n(\%)$} & \multicolumn{2}{|c|}{ Physical fatigue } & \multicolumn{2}{|c|}{ Mental fatigue } \\
\hline & & $\mathrm{M}(\mathrm{P} 25, \mathrm{P} 75)$ & $\begin{array}{c}\mathrm{Z}^{*} / \mathrm{H}^{* *} \\
P\end{array}$ & $\mathrm{M}(\mathrm{P} 25, \mathrm{P} 75)$ & $\begin{array}{c}\mathrm{Z}^{*} / \mathrm{H}^{* *} \\
P\end{array}$ \\
\hline \multicolumn{6}{|c|}{ The total number of days of rescue work (weeks) } \\
\hline$<1$ & $320(12.00)$ & $3(1,5)$ & $18.419^{* *}$ & $2(0,3)$ & $4.276^{* *}$ \\
\hline $1-2$ & $737(27.63)$ & $3(1,5)$ & 0.001 & $2(0,3)$ & 0.370 \\
\hline $3-4$ & $470(17.62)$ & $3(1,6)$ & & $1(0,3)$ & \\
\hline $5-6$ & $909(34.08)$ & $3(1,6)$ & & $2(0,3)$ & \\
\hline$\geq 7$ & $231(8.66)$ & $4(2,6)$ & & $1(0,3)$ & \\
\hline \multicolumn{6}{|c|}{ Average daily working hours (hours) } \\
\hline $4-6$ & $996(37.35)$ & $3(1,5)$ & $31.971^{* *}$ & $1(0,3)$ & $17.431^{* *}$ \\
\hline $7-8$ & $1230(46.12)$ & $3(1,6)$ & $<0.001$ & $1(0,3)$ & 0.001 \\
\hline $9-10$ & 349 (13.09) & $4(2,6)$ & & $2(1,3)$ & \\
\hline$\geq 11$ & $92(3.45)$ & $5(2,8)$ & & $2(1,4)$ & \\
\hline \multicolumn{6}{|c|}{ Frequency of weekly night shifts (times) } \\
\hline 0 & $837(31.38)$ & $3(1,6)$ & $29.002^{* *}$ & $1(0,3)$ & $18.467^{* *}$ \\
\hline 1 & $504(18.90)$ & $3(1,5)$ & $<0.001$ & $2(0,3)$ & 0.001 \\
\hline 2 & $867(32.51)$ & $3(1,6)$ & & $1(0,3)$ & \\
\hline 3 & $351(13.16)$ & $4(2,6)$ & & $2(1,3)$ & \\
\hline 4 & $108(4.05)$ & $4(2,7)$ & & $2(0,3)$ & \\
\hline \multicolumn{6}{|c|}{ Have you directly participated in the rescue of patients with COVID-19 } \\
\hline Yes & $742(27.82)$ & $4(2,6)$ & $-7.133^{*}$ & $2(0,3)$ & $-3.255^{*}$ \\
\hline No & $1925(72.18)$ & $3(1,5)$ & $<0.001$ & $1(0,3)$ & 0.001 \\
\hline \multicolumn{6}{|c|}{ Have you received professional protection training } \\
\hline Yes & $2487(93.25)$ & $3(1,6)$ & $-4.879^{*}$ & $1(0,3)$ & $-4.539^{*}$ \\
\hline No & $180(6.75)$ & $5(2,7)$ & $<0.001$ & $3(1,4)$ & $<0.001$ \\
\hline \multicolumn{6}{|c|}{ Have you received any professional psychological assistance } \\
\hline Yes & $274(10.27)$ & $2(1,5.25)$ & $-3.160^{*}$ & $2(0,3)$ & $-0.066^{*}$ \\
\hline No & $2393(89.73)$ & $3(1,6)$ & 0.002 & $1(0,3)$ & 0.947 \\
\hline \multicolumn{6}{|c|}{ Have you had occupational exposure } \\
\hline Yes & $260(9.75)$ & $5(2.25,7)$ & $-7.505^{*}$ & $2(1,3)$ & $-3.638^{*}$ \\
\hline No & $2407(90.25)$ & $3(1,6)$ & $<0.001$ & $1(0,3)$ & $<0.001$ \\
\hline \multicolumn{6}{|c|}{ Have you been infected with COVID-19 } \\
\hline Yes & $86(3.22)$ & $4.5(2.75,6)$ & $-3.239^{*}$ & $2(1,3)$ & $-1.222^{*}$ \\
\hline No & $2581(96.78)$ & $3(1,6)$ & 0.001 & $1(0,3)$ & 0.222 \\
\hline \multicolumn{6}{|c|}{ Have you had any experience of negative events } \\
\hline Yes & $646(24.22)$ & $4(2,7)$ & $-9.032^{*}$ & $2(0,3)$ & $-3.939^{*}$ \\
\hline No & $2021(75.78)$ & $3(1,5)$ & $<0.001$ & $1(0,3)$ & $<0.001$ \\
\hline \multicolumn{6}{|c|}{ Degree of fear with COVID-19 } \\
\hline None & $176(6.60)$ & $1(0,4)$ & $243.569^{* *}$ & $1(0,3)$ & $75.627^{* *}$ \\
\hline Mild & $729(27.33)$ & $2(0,4)$ & $<0.001$ & $1(0,3)$ & $<0.001$ \\
\hline Moderate & $1050(39.37)$ & $3(2,6)$ & & $2(0,3)$ & \\
\hline Severe & $608(22.80)$ & $4(2,7)$ & & $2(1,3)$ & \\
\hline Excessive severe & $104(3.90)$ & $6(3,8)$ & & $3(1,5)$ & \\
\hline
\end{tabular}

M: median; P25: percentile 25; P75: percentile $75 ;{ }^{*} \mathrm{Z}$ : Mann-Whitney test; ${ }^{* *} \mathrm{H}$ : Kruskal-Wallis test

observed between the average number of weekly exercise and nurses' fatigue $(\mathrm{B}=0.266 ; P<0.001)$. It was also found that average daily working hours had a significantly positive correlation with nurses' fatigue $(\mathrm{B}=0.270 ; P<0.001)$. Meanwhile, anxiety and perceived stress were also positively associated with nurses' fatigue, which means that anxiety $(\mathrm{B}=0.068 ; P=0.003)$ and perceived stress $(\mathrm{B}=0.103 ; P<0.001)$ increase the nurses' fatigue. In addition, the average night shift times every week had a low positive correlation with nurses' fatigue $(\mathrm{B}=0.070 ; P=0.036)$.

\section{DISCUSSION}

\subsection{Overall Fatigue State of First-line Nurses in Wuhan}

In our study over a third nurses had a substantial symptoms of tiredness among first-line nurses in Wuhan, accordant with the findings reported in study conducted in United States ${ }^{[27]}$. The result showed the incidence of fatigue was moderate, $35.06 \%$ nurses of the all participants had a total score of $\geq 7$, the median score was $10(8,11)$, the median score of physical 
Table 3 Descriptive and univariate analyses between fatigue and anxiety, depression and perceived stress variables $(n=2667)$

\begin{tabular}{|c|c|c|c|c|c|}
\hline \multirow{3}{*}{ Variables } & \multirow{3}{*}{$n(\%)$} & \multicolumn{2}{|c|}{ Physical fatigue } & \multicolumn{2}{|c|}{ Mental fatigue } \\
\hline & & \multirow{2}{*}{$\mathrm{M}(\mathrm{P} 25, \mathrm{P} 75)$} & Z & \multirow{2}{*}{$\mathrm{M}(\mathrm{P} 25, \mathrm{P} 75)$} & Z \\
\hline & & & $P$ & & $P$ \\
\hline \multicolumn{6}{|l|}{ Anxiety } \\
\hline Yes & $1062(39.81)$ & $6(4,8)$ & -27.655 & $3(1,4)$ & -20.648 \\
\hline No & $1605(60.16)$ & $2(0,3)$ & $<0.001$ & $1(0,2)$ & $<0.001$ \\
\hline \multicolumn{6}{|c|}{ Depression } \\
\hline Yes & $1458(54.65)$ & $5(3,7)$ & -30.020 & $2(1,4)$ & -19.678 \\
\hline No & 1209 (45.33) & $1(0,3)$ & $<0.001$ & $1(0,2)$ & $<0.001$ \\
\hline \multicolumn{6}{|c|}{ Perceived stress } \\
\hline Yes & $1298(62.00)$ & $5(3,7)$ & -22.485 & $3(1,4)$ & -24.001 \\
\hline No & $1369(38.00)$ & $2(0.5,4)$ & $<0.001$ & $1(0,2)$ & $<0.001$ \\
\hline
\end{tabular}

M: median; P25: percentile 25; P75: percentile 75; Z: Mann-Whitney test

Table 4 Bivariate correlations between fatigue and anxiety, depression and perceived stress variables of the nurses ( $n=2667)$

\begin{tabular}{llcccccc}
\hline Variables & & Anxiety & Depression & Perceived stress & Physical fatigue & Mental fatigue & Fatigue \\
\hline Anxiety & $r$ & 1.000 & 0.862 & 0.591 & 0.605 & 0.467 & 0.638 \\
& $P$ & - & 0.000 & 0.000 & 0.000 & 0.000 & 0.000 \\
Depression & $r$ & 0.862 & 1.000 & 0.578 & 0.653 & 0.493 & 0.683 \\
& $P$ & 0.000 & - & 0.000 & 0.000 & 0.000 & 0.000 \\
Perceived stress & $r$ & 0.591 & 0.578 & 1.000 & 0.515 & 0.495 & 0.586 \\
& $P$ & 0.000 & 0.000 & - & 0.000 & 0.000 & 0.000 \\
Physical fatigue & $r$ & 0.605 & 0.653 & 0.515 & 1.000 & 0.471 & 0.921 \\
& $P$ & 0.000 & 0.000 & 0.000 & - & 0.000 & 0.000 \\
Mental fatigue & $r$ & 0.467 & 0.493 & 0.495 & 0.471 & 1.000 & 0.777 \\
& $P$ & 0.000 & 0.000 & 0.000 & 0.000 & - & 0.000 \\
Fatigue & $r$ & 0.638 & 0.683 & 0.586 & 0.921 & 0.777 & 1.000 \\
& $P$ & 0.000 & 0.000 & 0.000 & 0.000 & 0.000 & -
\end{tabular}

Table 5 Multiple linear regression of fatigue and work-related factors, anxiety, depression, perceived stress variables of the nurses $(n=2667)$

\begin{tabular}{|c|c|c|c|c|c|c|c|}
\hline \multirow{2}{*}{ Variables } & \multicolumn{2}{|c|}{ Regression coefficients } & \multirow{2}{*}{$\frac{\text { Standardized coefficients }}{B e t a}$} & \multirow{2}{*}{ 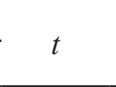 } & \multirow{2}{*}{$P$} & \multicolumn{2}{|c|}{$95 \%$ Confidence interval } \\
\hline & $B$ & Standard error & & & & Lower limit & Upper limit \\
\hline Constant & 1.345 & 0.839 & - & 1.602 & 0.109 & -0.301 & 2.990 \\
\hline Average length of daily break & -0.053 & 0.058 & -0.012 & -0.905 & 0.365 & -0.168 & 0.062 \\
\hline Average length of daily night sleep & -0.031 & 0.174 & -0.002 & -0.180 & 0.857 & -0.373 & 0.310 \\
\hline Average number of weekly exercise & -0.266 & 0.070 & -0.052 & -3.809 & 0.000 & -0.403 & -0.129 \\
\hline $\begin{array}{l}\text { Direct participation in the rescue of } \\
\text { patients with COVID-19 }\end{array}$ & -0.189 & 0.114 & -0.022 & -1.654 & 0.098 & -0.412 & 0.035 \\
\hline Average daily working hours & 0.270 & 0.065 & 0.056 & 4.148 & 0.000 & 0.142 & 0.397 \\
\hline Average number of weekly night shifts & 0.070 & 0.033 & 0.029 & 2.096 & 0.036 & 0.004 & 0.135 \\
\hline Professional protection training & 0.145 & 0.203 & 0.010 & 0.715 & 0.475 & -0.252 & 0.542 \\
\hline Occupational exposure & -0.295 & 0.173 & -0.023 & -1.700 & 0.089 & -0.634 & 0.045 \\
\hline Being infected with COVID-19 & -0.274 & 0.288 & -0.013 & -0.951 & 0.342 & -0.839 & 0.291 \\
\hline Experience of negative events & 0.186 & 0.120 & 0.021 & 1.549 & 0.122 & -0.049 & 0.421 \\
\hline Degree of fear with COVID-19 & -0.038 & 0.057 & -0.010 & -0.670 & 0.503 & -0.151 & 0.074 \\
\hline Anxiety & 0.068 & 0.023 & 0.081 & 2.961 & 0.003 & 0.023 & 0.113 \\
\hline Depression & 0.303 & 0.018 & 0.440 & 16.411 & 0.000 & 0.267 & 0.339 \\
\hline Perceived Stress & 0.130 & 0.008 & 0.267 & 15.688 & 0.000 & 0.114 & 0.147 \\
\hline
\end{tabular}

and mental fatigue of them were $7(5,8)$ and $3(2,4)$ respectively. During the acute SARS outbreak, $89 \%$ of health care workers who were in high-risk situations reported psychological symptoms. Nurses' fatigue has been recognized as a threat to nurses' health and patient safety ${ }^{[12]}$. Fatigue is not only a status, but also a process.
It has been reported that the incidence of fatigue is $35.3 \%-50.00 \%$ in the recent relative studies ${ }^{[27,28]}$. If we do not pay attention to the reasonable arrangement of rest, nurses are prone to fatigue syndrome. Considering the extremely high risk of tiredness symptom among first-line health care workers in Wuhan, their physical 
and mental health may require special attention.

3.2 Analysis on Influencing Factors of Fatigue of First-line Nurses in Wuhan

\subsubsection{Daily Working Hours As revealed in the} multiple linear regression models of fatigue, average daily working hours had a significantly positive correlation with nurses' fatigue. It has been proved that extended working hours result in the escalating exhaustion, increased traumas and decreased nursing actions $s^{[8,9]}$. In our study, we found that the mean score of nurses with daily working hours of 4-6 was the lowest. On the contrary, in groups of 8-10 hours and 10-12 hours, the mean score was higher. Engaging in the rescue nursing work of patients with COVID-19 was a series of challenges. Due to the particularity of the pandemic, close use of the protective clothes, glasses and N95 masks for a long time were prone to increase physical discomfort, such as pressure injury, difficulty in nursing practice, even hard to guarantee physiological needs, resulting in physical consumption and affecting work efficiency to a certain extent. When nurses continued to struggle with the pandemic, as the working hours were prolonged and volatile, nurses' psychological states would constantly be disturbed by external work, and the physical fatigue of nurses might also be affected by the continuous efforts in the front line. Meanwhile, mental fatigue has a certain impact, and eventually becomes a major hidden risk for safe nursing work.

3.2.2 Frequency of Night Shift The finding of present study showed that the frequency of weekly night shift had a low positive correlation with nurses' fatigue. The previous study provided evidence supporting the association of work shift length with fatigue $^{[29]}$. It was found that night shift of 3-4 times per week indicated much higher level of fatigue, and the more frequent night shift, the higher fatigue level in our study. Nursing work is both physically and mentally laborious. In the earlier stage of the pandemic, most nurses from different departments were dispatched to the isolation wards urgently, plunged into rescue work with immense obligation, accompanied by the higher frequency of night shift with $8 \mathrm{~h}$ in the initial period, consecutive works with insufficient rest during the night on-duty resulted in the physical and mental burnout. Sagherian et al[30] gave a strong evidence that in nurses who experienced shift work schedules, working long hours, disturbed circadian rhythm, fatigue eventually became unavoidable and carrying out optimal nursing performance was a challenge. Based on our findings, it was also found after decreased the frequency of night shift to 1-2 times per week through effective managements and other creative and innovative workplace scheduling, the level of nursers' fatigue declined significantly.

3.2.3 Lifestyle Factors This study reported that the frequency of exercise and nurses' fatigue had a statistically significant negative correlation, and nurses without taking any exercises showed higher level of physical fatigue than in the exercise groups. Healthy lifestyle can effectively improve the process of nurses' health promotion and physical activity or exercise had been proved to be linked with an increase in sleep quality and to reduce chronic fatigue ${ }^{[31]}$. Despite other lifestyle variables did not enter the multivariate analysis lastly, the subsequent lifestyle-related variables which were statistically significant in univariate analyses needed to be noticed, for nurses in groups of 30-60 min and over $60 \mathrm{~min}$ for daily lunch break, and nurses with sufficient night sleep, showed lower scores than in the group without lunch break and adequate night sleep. Previous study indicated a significant relationship between insufficient rest period and fatigue ${ }^{[30]}$. The intense rescue work and shortage of human resources can lead to the repeated restriction of rest, substantially impair cognitive performance and decrease alertness among the first-line nurses, resulting in the distinctly greater level of fatigue.

3.2.4 Psychological Factors Psychological health related factors were included in our study. At present, a series of studies ${ }^{[32-34]}$ about mental health research of rescue nurses in Wuhan indicated that nurses experienced continuous and aggravated negative psychological problems in many aspects, including anxiety, depression and stress, etc., which were consistent with the results in this study. In the present study, the risk symptoms of anxiety, depression and perceived stress in nurses had significant influences on physical and mental fatigue. The higher level of anxiety, depression and perceived stress existed, the more serious degree of physical and mental fatigue emerged. A statistically significant positive correlation was observed between the variables and fatigue through Pearson's correlation analysis $(P<0.01)$. Our results were consistent with those in the previous studies that fatigue was associated with levels of anxiety and depression ${ }^{[35,36]}$. Most first-line nurses are females, whose personality characteristics are sensitive, fragile and prone to psychological crises such as insecurity and fear, which leads to aggravation of fatigue. In addition, some first-line nurses are lack of experience in infectious disease rescue and emergency care technology, which also brings great psychological stress.

\subsection{Intervention Strategies for Fatigue of First-line Rescue Nurses}

There was a moderate level of fatigue among the first-line nurses fighting against COVID-19 pandemic in Wuhan, China. Individuals, health authorities and government need to formulate and take effective intervention strategies according to the relevant risk factors, and undertake preventive measures aimed at reducing health hazards due to increased work- 
related fatigue among first-line nurses, and to enhance their health status and provide a safe occupational environment worldwide.

\subsubsection{Individual System Fatigue has a detrimental} effect on nurses' overall perceptions of patient safety ${ }^{[37]}$. Work-related fatigue includes physiological, cognitive, emotional and sensory elements that result as a consequence of high work volume and insufficient time for energy recovery. The physical and mental fatigue is likely present among nurses. Physical fatigue is caused by physical labor and load, which is characterized by general discomfort and reduced ability to produce strength or power. Mental fatigue is caused by mental task and stress, resulting in elevated level of fatigue and decreased level of concentration, motivation and alertness ${ }^{[38]}$. The persistence in healthy lifestyle is encouraged, moderate exercise is conducive to relieve the state of nervous tension, eliminate tension, release psychological pressure, promote deep sleep, and optimal rest ${ }^{[32,31]}$. Methods for nurses to deal with psychological crisis or emotional response, such as appropriate emotional catharsis allow themselves to have negative emotions, and detect and adjust them timely; find suitable relaxation ways; change cognition and attitude; maintain full understanding between nurses and patients; seek social support when necessary; share their feelings and experiences with colleagues and peers; choose to keep in touch with family and friends; increase the family background resources; make up for the lack of resources brought by high work family conflict, in order to improve work passion and alleviate psychological problems, maintain physical and mental integrity and activeness.

\subsubsection{Health Care System The complexity and} multidimensional nature of nursing work may lead to fatigue, and health care institutions need to better understand the factors that contribute to nurses' fatigue and consider accurate mitigation strategies. Fatigue Risk Management Systems (FRMS) ${ }^{[39]}$ was recommended to set up in hospitals to provide a comprehensive approach to reduce risks from fatigue, including instituting workplace policies, establishing procedures to protect tasks that are vulnerable to fatigue-related errors, promoting education for managers and nurses, taking fatigue-related factors into the considerations of investigation on adverse events. The hospital officials should ensure a better working environment for medical staff, appropriate working hours and frequency of night shift, eliminate job burnout, improve the work immersion level of nurses, and reduce the occurrence of adverse events. It is suggested that countries and regions with the pandemic should provide strong support for rescue nurses to lessen work-related fatigue in daily working. It is necessary to request the support of superior departments, allocate the human resources of nurses reasonably, according to the dynamic and flexible principle, adjust the working mode and working length according to the workload, and reduce unnecessary too-prolonged work for first-line nurses.

\subsubsection{Government System Every person and} each organization are involved in the rescue work when encountering the pandemic. Government should establish and improve the response mechanism for major public health emergencies ${ }^{[6]}$, and give full play to the role of the government system in each region combating with the pandemic, concentrate and integrate the advantageous resources, establish a complete medical and life support system, and provide strong social support for the medical staff and organizations participating in current rescue. Providing humanistic care to all the rescue staff, including solving the necessary problems of dining, accommodation, life, transportation, so as to ensure their strong enforce to participate in and promote the improvement of team performance while reducing the conflict experience, may be beneficial for relieving their negative mental stress. The organization should provide support on mental health knowledge with the aim of mastering the psychological stress reaction process and performance of nurses appropriately, implement targeted mental health assessment and intervention, strengthen the psychological crisis intervention during and after the pandemic situation, which could cut down the negative emotions of medical staff effectively.

\subsection{Limitations of Study}

The present research has several limitations. First, a descriptive cross-sectional design was used, and no cause-and-effect relationships were established. Thus, it would be necessary to conduct a longitudinal study and ascertain the variables with a cause-effect relationship. Secondly, the study was based on a questionnaire survey with self-report instruments, and all of the variables were measured in terms of participants' subjective perceptions, the bias is not avoidable, which can affect the response reliability. Future studies using random sampling frames can enhance the external validity of the findings. Nevertheless, we made efforts to control for the personal and professional characteristics of participants to provide the clearest possible image of working as first-line nurses combating with COVID-19 in Wuhan, China. Further prudent investigation using multiple modes of inquiry should be performed and deeper interviews during periodical examinations may be needed on this topic to diagnose chronic fatigue syndrome.

\subsection{Implications for Future Research and Practice}

This study is of great significance to the rescue nursing work of the pandemic currently. First, health care authorities should be aware of this issue and pay more attention to the physical and mental state of nurses, for it could lead to adverse health conditions for nurses working in current severe situation of pandemic 
prevention and control. The long-term fatigue of nurses not only damages individual physical and mental health, increases psychological crisis events for nurses under the rescue condition, but also reduces the efficiency and quality of rescue work, patients' disease recovery and safety might be affected. It is imperative to strengthen the support and guarantee system of national government and health care settings for rescue nurses all over the world.

To sum up, addressing fatigue and managing risk across industries have received growing attention in the occupational health literature. While fatigue in nurses is a significant challenge in health care, concrete guidelines and methods to reduce fatigue or mitigate its negative effects on safety and performance are lacking. Findings from the present research could promote more comprehensive awareness of the contributing factors in the rescue work-related system and psychological variables relate to increased fatigue levels, ultimately guide the design of relevant and appropriate fatigue mitigation interventions, and undertake measures to lessen fatigue in practice context of combating with the pandemic at present.

\section{Acknowledgement}

Authors acknowledge all participants who responded to the surveys and the nursing administrators for the cooperation with their effort and time in conducting the present study.

\section{Conflict of Interest Statement}

The authors declare that there is no conflict of interest regarding the publication of this article.

\section{REFERENCES}

1 Aaronson LS, Teel CS, Cassmeyer V, et al. Defining and Measuring Fatigue. J Nurs Scholarship, 1999,31(1):4550

2 Smith-Miller CA, Shaw-Kokot J, Curro B, et al. An Integrative Review Fatigue Among Nurses in Acute Care Settings. J Nurs Admin, 2014,44(9):487-494

3 Steege LM, Dykstra JG. A macroergonomic perspective on fatigue and coping in the hospital nurse work system. Appl Ergon, 2016,54:19-26

4 Kahriman İ, Öztürk H. Evaluating medical errors made by nurses during their diagnosis, treatment and care practices. J Clin Nurs, 2016,25(19-20):2884-2894

5 Li Q, Guan X, Wu P, et al. Early Transmission Dynamics in Wuhan, China, of Novel Coronavirus-Infected Pneumonia. N Engl J Med, 2020,382(13):1199-1207

6 Organization WH. Coronavirus disease 2019 (COVID-19). Situation Report-50. https://www.who.int/docs/defaultsource/coronaviruse/situation-reports/20200310-sitrep50 -covid-19.pdf?sfvrsn $=55 \mathrm{e} 904 \mathrm{fb} \_2$

7 Lai J, Ma S, Wang Y, et al. Factors Associated With Mental Health Outcomes Among Health Care Workers Exposed to Coronavirus Disease 2019. JAMA Netw Open, 2020,3(3):e203976

8 Jones G, Hocine M, Jérôme Salomon, et al. Demographic and occupational predictors of stress and fatigue in French intensive-care registered nurses and nurse's aides: A cross-sectional study. Int J Nurs Study, 2014,52(1):250-259

9 Samaha E, Lal S, Samaha N, et al. Psychological, lifestyle and coping contributors to chronic fatigue in shift-worker nurses. J Adv Nurs, 2007,59(1):221-232

10 Kawano, Yuri. Association of Job-related Stress Factors with Psychological and Somatic Symptoms among Japanese Hospital Nurses: Effect of Departmental Environment in Acute Care Hospitals. J Occup Health, 2008,50(1):79-85

11 Maunder R, Hunter J, Vincent L, et al. The immediate psychological and occupational impact of the 2003 SARS outbreak in a teaching hospital. CMAJ, 2003, 168(10):1245-1251

12 Bai Y, Lin CC, Lin CY, et al. Survey of stress reactions among health care workers involved with the SARS outbreak. Psychiatr Serv, 2004,55(9):1055-1057

13 Lee AM, Wong JG, McAlonan GM, et al. Stress and psychological distress among SARS survivors 1 year after the outbreak. Can J Psychiatry, 2007,52(4):233240

14 Lee ML, Chen CJ, Su IJ, et al. Severe acute respiratory syndrome--Taiwan, 2003, MMWR Morb Mortal Wkly Rep, 2003, 52(20):461-466

15 Liu C, Wang H, Zhou L, et al. Sources and symptoms of stress among nurses in the first Chinese anti-Ebola medical team during the Sierra Leone aid mission: A qualitative study. Int J Nurs Sci, 2019,6(2):187-191

16 Lehmann M, Bruenahl CA, Löwe B, et al. Ebola and Psychological Stress of Health Care Professionals. Emerg Infect Dis, 2015,21(5):913-914

17 Taylor-East R, Grech A, Gatt C. The mental health of newly graduated doctors in Malta. Psychiatr Danub, 2013,25(2):250-255

18 Morriss RK, Wearden AJ, Mullis R. Exploring the validity of the Chalder Fatigue scale in chronic fatigue syndrome. J Psychosom Res, 1998,45(5):411-417

19 Spitzer RL, Kroenke K, Williams JBW, et al. A Brief Measure for Assessing Generalized Anxiety Disorder: The GAD-7. Arch Intern Med, 2006,166(10):1092-1097

20 Schalet BD, Cook KF, Choi SW, et al. Establishing a common metric for self-reported anxiety: Linking the MASQ, PANAS, and GAD-7 to PROMIS Anxiety. J Anxiety Disord, 2014,28(1):88-96

21 He XY, Li CB, Qian J, et al. Reliability and validity of a generalized anxiety scale in general hospital outpatients. Shanghai Arch Psychiatry, 2010,22(4):200-203

22 Manea L, Gilbody S, Mcmillan D. A diagnostic metaanalysis of the Patient Health Questionnaire-9 (PHQ-9) algorithm scoring method as a screen for depression. Gen Hosp Psychiatry, 2015,37(1):67-75

23 Kroenke K, Spitzer RL, Williams JBW. The PHQ-9: Validity of a Brief Depression Severity Measure. J Gen Intern Med, 2001,16(9):606-613

24 Bian CD, He XY, Qian J, et al. Study on the application of depression symptom cluster scale of patient health questionnaire in general hospitals. J Tongji Univ (Med Sci) (Chinese), 2009,30(5):136-140

25 Yang TZ, Huang HT. An epidemiological study on the psychological stress of urban residents in social transformation. Chin J Epidemiol (Chinese), 2003, 
24(9):760-764

26 Shen J, Yu H, Zhang Y, et al. Professional quality of life: A cross-sectional survey among Chinese clinical nurses. Nurs Health Sci, 2015,17(4):507-515

27 Dyrbye LN, Shanafelt TD, Johnson PO, et al. A crosssectional study exploring the relationship between burnout, absenteeism, and job performance among American nurses. BMC Nurs, 2019,18:57-65

28 Zdanowicz $\mathrm{T}$, Turowski $\mathrm{K}$, Celej-Szuster $\mathrm{J}$, et al. Insomnia, Sleepiness, and Fatigue Among Polish Nurses. Workplace Health Saf, 2020,68(6):272-278

29 Ferri P, Guadi M, Marcheselli L, et al. The impact of shift work on the psychological and physical health of nurses in a general hospital: a comparison between rotating night shifts and day shifts. Risk Manag Healthc Policy, 2016,14(9):203-211

30 Sagherian K, Clinton ME, Huijer AS, et al. Fatigue, Work Schedules, and Perceived Performance in Bedside Care Nurses. Workplace Health Saf, 2017,65(7):304312

31 Atkinson PG, Fullick S, Grindey C, et al. Exercise, Energy Balance and the Shift Worker. Sports Med, 2008,38(8):671-685

$32 \mathrm{Xu} \mathrm{MC}$, Zhang Y. Investigation on the psychological status of the first clinical first-line support nurses against novel coronavirus pneumonie. Chin Nurs Res (Chinese), 2020,34(3):368-370

$33 \mathrm{Li}$ CS, Mi YY, Chu J, et al. Investigation and analysis of novel coronavirus first-line caregivers with posttraumatic stress disorder. J Nurses Training (Chinese), 2020,35(07):615-618

34 Jiang X, Tan X. Investigation on the mental health status of first line nurses during the outbreak of COVID-19. J Nurs Sci (Chinese), 2020,35(07):75-77

35 Çelik S, Taşdemir N, Kurt A, et al. Fatigue in Intensive Care Nurses and Related Factors. Int J Occup Environ Med, 2017,88(4):199-206

36 Jones G, Hocine M, Salomon J, et al. Demographic and occupational predictors of stress and fatigue in French intensive-care registered nurses and nurses' aides: A cross-sectional study. Int J Nurs Stud, 2015,52(1):250259

37 Qasim AM, Loai AS, Omar AO. Fatigue, burnout, work environment, workload and perceived patient safety culture among critical care nurses. Br J Nurs, 2020,29(1):28-34

38 Pasupathy KS, Barker LM. Impact of fatigue on performance in registered nurses: Data mining and implications for practice. J Healthc Qual, 2011,34(5):2230

39 Steege L, Pinekenstein B, Rainbow J, et al. Addressing occupational fatigue in nurses: current state of fatigue risk management in hospitals, part 1. J Nurs Adm, 2017,46(4):426-433

(Received Apr. 3, 2020; accepted July 12, 2020) 